TRANSACTIONS OF THE

AMERICAN MATHEMATICAL SOCIETY

Volume 358, Number 1, Pages 215-232

S 0002-9947(04)03623-2

Article electronically published on December 28, 2004

\title{
SYMMETRIC FUNCTIONS IN NONCOMMUTING VARIABLES
}

\author{
MERCEDES H. ROSAS AND BRUCE E. SAGAN
}

\begin{abstract}
Consider the algebra $\mathbb{Q}\left\langle\left\langle x_{1}, x_{2}, \ldots\right\rangle\right\rangle$ of formal power series in countably many noncommuting variables over the rationals. The subalgebra $\Pi\left(x_{1}, x_{2}, \ldots\right)$ of symmetric functions in noncommuting variables consists of all elements invariant under permutation of the variables and of bounded degree. We develop a theory of such functions analogous to the ordinary theory of symmetric functions. In particular, we define analogs of the monomial, power sum, elementary, complete homogeneous, and Schur symmetric functions as well as investigating their properties.
\end{abstract}

\section{INTRODUCTION}

Let $\mathbb{Q}\left[\left[x_{1}, x_{2}, \ldots\right]\right]=\mathbb{Q}[[\mathbf{x}]]$ be the algebra of formal power series over $\mathbb{Q}$ in a countably infinite set of commuting variables $x_{i}$. For each positive integer $m$, the symmetric group $\mathfrak{S}_{m}$ acts on $\mathbb{Q}[[\mathbf{x}]]$ by

$$
g f\left(x_{1}, x_{2}, \ldots\right)=f\left(x_{g(1)}, x_{g(2)}, \ldots\right),
$$

where $g(i)=i$ for $i>m$. We say that $f$ is symmetric if it is invariant under the action of $\mathfrak{S}_{m}$ for all $m \geq 1$. The algebra of symmetric functions, $\Lambda=\Lambda(\mathbf{x})$, consists of all symmetric $f$ of bounded degree. This algebra has a long, venerable history in combinatorics, algebraic geometry, and representation theory; see, e.g., [6, 18, 26, 32.

Now consider $\mathbb{Q}\left\langle\left\langle x_{1}, x_{2}, \ldots\right\rangle\right\rangle=\mathbb{Q}\langle\langle\mathbf{x}\rangle\rangle$, the associative algebra of formal power series in the noncommuting variables $x_{1}, x_{2}, \ldots$. Define the algebra of symmetric functions in noncommuting variables, $\Pi=\Pi(\mathbf{x})$, to be the subalgebra consisting of all elements in $\mathbb{Q}\langle\langle\mathbf{x}\rangle\rangle$ which are invariant under the action defined by (1) and of bounded degree. (This is not to be confused with the algebra of noncommutative symmetric functions of Gelfand et al. 9] or the partially commutative symmetric functions studied by Lascoux and Schützenberger [15] as well as by Fomin and Greene [5].) This algebra was first studied by M. C. Wolf [36] in 1936. Her goal was to provide an analogue of the fundamental theorem of symmetric functions in this context. The concept then lay dormant for over 30 years until Bergman and Cohn generalized Wolf's result [2]. Still later, Kharchenko [13] proved that if $V$ is a graded vector space and $G$ a group of grading-preserving automorphisms of the tensor algebra of $V$, then the algebra of invariants of $G$ is also a tensor algebra. Anick [1] then removed the condition that $G$ preserve the grading. Most recently,

Received by the editors October 26, 2002 and, in revised form, January 30, 2004.

2000 Mathematics Subject Classification. Primary 05E05; Secondary 05E10, 05A18.

Key words and phrases. Noncommuting variables, partition lattice, Schur function, symmetric function. 
Gebhard and Sagan [8] revived these ideas as a tool for studying Stanley's chromatic symmetric function of a graph [29, 31].

This paper gives the first systematic study of $\Pi(\mathbf{x})$ and is structured as follows. In the next section we define $\Pi(\mathbf{x})$-analogues for the monomial, power sum, elementary, and complete homogeneous bases of $\Lambda(\mathbf{x})$. We relate the two sets of bases in Theorem 2.1 using the projection map $\rho: \mathbb{Q}\langle\langle\mathbf{x}\rangle\rangle \rightarrow \mathbb{Q}[[\mathbf{x}]]$ which lets the variables commute. In Section 3 we derive change of basis equations for these four bases by summation or Möbius inversion over the lattice of partitions. As a consequence, we obtain some properties of the fundamental involution $\omega: \Pi(\mathbf{x}) \rightarrow \Pi(\mathbf{x})$ in Theorem 3.5. In the following section, we define a right inverse for $\rho$, called the lifting map, and study its relation with an inner product on $\Pi(\mathbf{x})$. In Section 5 we recall some facts about the algebra of MacMahon symmetric functions $\mathcal{M}$ and show that a particular subspace of $\mathcal{M}$ is naturally isomorphic to $\Pi(\mathbf{x})$ as a vector space. This permits us to define a noncommuting-variable analogue, $S_{\lambda}$, of a Schur function in Section 6. The next two sections are devoted to obtaining analogues for $S_{\lambda}$ of the Jacobi-Trudi determinants (Theorem 7.1) and Robinson-Schensted-Knuth algorithm (Theorem 8.1). We end with a list of comments and open questions.

\section{BASIC DEFINITIONS}

Let $n$ be a nonnegative integer. For $\lambda=\left(\lambda_{1}, \lambda_{2}, \ldots, \lambda_{l}\right)$ a partition of $n$, we write $\lambda \vdash n$ and denote the length of $\lambda$ by $l=l(\lambda)$. We will use the notation

$$
\lambda=\left(1^{m_{1}}, 2^{m_{2}}, \ldots, n^{m_{n}}\right)
$$

to mean that $i$ appears in $\lambda$ with multiplicity $m_{i}, 1 \leq i \leq n$. The bases of the symmetric function algebra $\Lambda(\mathbf{x})$ are indexed by partitions. Following [18, 32, we use the notation $m_{\lambda}, p_{\lambda}, e_{\lambda}$, and $h_{\lambda}$ for the monomial, power sum, elementary, and complete homogeneous symmetric functions bases. Our next goal is to define the analogues of these bases in noncommuting variables; these analogues will be labeled by set partitions.

Define $[n]=\{1,2, \ldots, n\}$. A set partition $\pi$ of $[n]$ is a family of disjoint sets, called blocks $B_{1}, B_{2}, \ldots, B_{l}$, whose union is $[n]$. We write $\pi=B_{1} / B_{2} / \ldots / B_{l} \vdash[n]$ and define length $l=l(\pi)$ as the number of blocks. There is a natural mapping from set partitions to integer partitions given by

$$
\lambda(\pi)=\lambda\left(B_{1} / B_{2} / \cdots / B_{l}\right)=\left(\left|B_{1}\right|,\left|B_{2}\right|, \ldots,\left|B_{l}\right|\right),
$$

where we assume that $\left|B_{1}\right| \geq\left|B_{2}\right| \geq \cdots \geq\left|B_{l}\right|$. The integer partition $\lambda(\pi)$ is the type of the set partition $\pi$.

The partitions of $[n]$ form the partition lattice $\Pi_{n}$. (Do not confuse $\Pi_{n}$ with the algebra $\Pi(\mathbf{x})$.) In $\Pi_{n}$ the ordering is by refinement: $\pi \leq \sigma$ if each block $B$ of $\pi$ is contained in some block $C$ of $\sigma$. The meet (greatest lower bound) and join (least upper bound) operations in $\Pi_{n}$ will be denoted $\wedge$ and $\vee$, respectively. There is a rank function in $\Pi_{n}$ given by $r(\pi)=n-l(\pi)$.

To obtain analogues of the bases of $\Lambda(\mathbf{x})$ in this setting, it will be helpful to think of the elements of $[n]$ as indexing the positions in a monomial $x_{i_{1}} x_{i_{2}} \cdots x_{i_{n}}$. This makes sense because the variables do not commute. Now given $\pi \vdash[n[$, define 
the monomial symmetric function, $m_{\pi}$ in noncommuting variables by

$$
m_{\pi}=\sum_{\left(i_{1}, i_{2}, \ldots, i_{n}\right)} x_{i_{1}} x_{i_{2}} \cdots x_{i_{n}} \quad \begin{aligned}
& \text { where the sum is over all } n \text {-tuples }\left(i_{1}, i_{2}, \ldots, i_{n}\right) \\
& \text { with } i_{j}=i_{k} \text { if and only if } j, k \text { are in the same } \\
& \text { block in } \pi .
\end{aligned}
$$

For example,

$m_{13 / 24}=x_{1} x_{2} x_{1} x_{2}+x_{2} x_{1} x_{2} x_{1}+x_{1} x_{3} x_{1} x_{3}+x_{3} x_{1} x_{3} x_{1}+x_{2} x_{3} x_{2} x_{3}+x_{3} x_{2} x_{3} x_{2}+\cdots$.

These functions are precisely the symmetrizations of monomials and so they are invariant under the action of $\mathfrak{S}_{m}$ defined previously. It follows easily that they form a basis for $\Pi(\mathbf{x})$.

We define the power sum function in noncommuting variables, $p_{\pi}$, by

$$
p_{\pi}=\sum_{\left(i_{1}, i_{2}, \ldots, i_{n}\right)} x_{i_{1}} x_{i_{2}} \cdots x_{i_{n}}, \text { where } i_{j}=i_{k} \text { if } j, k \text { are in the same block in } \pi \text {. }
$$

To illustrate,

$$
p_{13 / 24}=x_{1} x_{2} x_{1} x_{2}+x_{2} x_{1} x_{2} x_{1}+x_{1}^{4}+x_{2}^{4}+\cdots=m_{13 / 24}+m_{1234} .
$$

The elementary symmetric function in noncommuting variables is

$e_{\pi}=\sum_{\left(i_{1}, i_{2}, \ldots, i_{n}\right)} x_{i_{1}} x_{i_{2}} \cdots x_{i_{n}}$, where $i_{j} \neq i_{k}$ if $j, k$ are in the same block in $\pi$.

By way of example,

$$
\begin{aligned}
e_{13 / 24} & =x_{1} x_{1} x_{2} x_{2}+x_{2} x_{2} x_{1} x_{1}+x_{1} x_{2} x_{2} x_{1}+x_{2} x_{1} x_{2} x_{1}+\cdots \\
& =m_{12 / 34}+m_{14 / 23}+m_{12 / 3 / 4}+m_{14 / 2 / 3}+m_{1 / 23 / 4}+m_{1 / 2 / 34}+m_{1 / 2 / 3 / 4} .
\end{aligned}
$$

To define the analogue of the complete homogeneous symmetric functions, it will be useful to introduce another way of looking at the previous definitions. (This was the method that Doubilet [4] used to define certain ordinary symmetric functions associated with set partitions.) Any two sets $D, R$ and a function $f: D \rightarrow R$ determine a kernel set partition, ker $f \vdash D$, whose blocks are the nonempty preimages $f^{-1}(r)$ for $r \in R$. For $f:[n] \rightarrow \mathbf{x}$ we denote by $M_{f}$ the corresponding monomial

$$
M_{f}=f(1) f(2) \cdots f(n) .
$$

Directly from these definitions it follows that

$$
m_{\pi}=\sum_{\operatorname{ker} f=\pi} M_{f}
$$

Using our running example, if $\pi=13 / 24$, then the functions with ker $f=\pi$ are exactly those of the form $f(1)=f(3)=x_{i}$ and $f(2)=f(4)=x_{j}$, where $i \neq j$. This $f$ gives rise to the monomial $M_{f}=x_{i} x_{j} x_{i} x_{j}$ in the sum for $m_{13 / 24}$.

Now define

$$
h_{\pi}=\sum_{(f, L)} M_{f}
$$

where $f:[n] \rightarrow \mathbf{x}$ and $L$ is a linear ordering of the elements of each block of $(\operatorname{ker} f) \wedge \pi$. Continuing with our running example,

$$
\begin{aligned}
& h_{13 / 24}=m_{1 / 2 / 3 / 4}+m_{12 / 3 / 4}+2 m_{13 / 2 / 4}+m_{14 / 2 / 3}+m_{1 / 23 / 4}+2 m_{1 / 24 / 3} \\
& \quad+m_{1 / 2 / 34}+m_{12 / 34}+4 m_{13 / 24}+m_{14 / 23}+2 m_{123 / 4}+2 m_{124 / 3}+2 m_{134 / 2} \\
& \quad+2 m_{1 / 234}+4 m_{1234} .
\end{aligned}
$$


Now we would like to give some justification to the above nomenclature by exhibiting its relation to that used for the ordinary symmetric functions. To this end, consider the projection map

$$
\rho: \mathbb{Q}\langle\langle\mathbf{x}\rangle\rangle \rightarrow \mathbb{Q}[[\mathbf{x}]]
$$

which merely lets the variables commute. We will need the notation

$$
\begin{aligned}
\lambda ! & =\lambda_{1} ! \lambda_{2} ! \cdots \lambda_{l} !, \\
\lambda^{!} & =m_{1} ! m_{2} ! \cdots m_{n} !,
\end{aligned}
$$

where the $m_{i}$ are the multiplicities in (2). We extend these conventions to set partitions by letting $\pi !=\lambda(\pi)$ ! and $\pi^{!}=\lambda(\pi)^{!}$. Note that

$$
\left(\begin{array}{l}
n \\
\lambda
\end{array}\right):=\text { number of } \pi \text { of type } \lambda=\frac{n !}{\lambda ! \lambda !} .
$$

The next proposition was proved by Doubilet for his set partition analogues of ordinary symmetric functions, and a similar proof can be given in the noncommuting case. The alternative demonstration given below brings out the combinatorics behind some of Doubilet's algebraic manipulations.

Theorem 2.1. The images of our bases under the projection map are:

(i) $\rho\left(m_{\pi}\right)=\pi ! m_{\lambda(\pi)}$,

(ii) $\rho\left(p_{\pi}\right)=p_{\lambda(\pi)}$,

(iii) $\rho\left(e_{\pi}\right)=\pi ! e_{\lambda(\pi)}$,

(iv) $\rho\left(h_{\pi}\right)=\pi ! h_{\lambda(\pi)}$.

Proof. For (i), let $B_{1}, B_{2}, \ldots, B_{k}$ be all blocks of $\pi$ of a given size. Recalling the remarks immediately before the definition of $m_{\pi}$, we see that $m_{\pi}$ is constant on the positions indexed by each of these $B_{i}$. Since $B_{1}, B_{2}, \ldots, B_{k}$ are of the same size, the variables in the positions indexed by $B_{i}$ can be interchanged with the variables in the positions indexed by $B_{j}$ for $1 \leq i, j \leq k$ to give another monomial in the sum for $m_{\pi}$ which maps to the same monomial in the projection. It follows that these blocks give rise to a factor of $k$ ! in the projection, and so $\pi$ will contribute $\pi$ !

To prove (ii), note that $p_{[n]}=m_{[n]}$ and so, from (i), $\rho\left(p_{[n]}\right)=m_{n}=p_{n}$. Now $p_{\lambda}=p_{\lambda_{1}} p_{\lambda_{2}} \cdots p_{\lambda_{l}}$. Furthermore, $p_{\pi}=p_{B_{1} / B_{2} / \cdots / B_{l}}$ is precisely the shuffle of $p_{\left[\lambda_{1}\right]}$, $p_{\left[\lambda_{2}\right]}, \ldots, p_{\left[\lambda_{l}\right]}$, where $\left|B_{i}\right|=\lambda_{i}$ and the elements from $p_{\left[\lambda_{i}\right]}$ are only permitted to be in the positions indexed by $B_{i}$. (We are letting the shuffle operation distribute over addition.) The desired equality follows.

The proof of (iii) is similar. We have $\rho\left(e_{[n]}\right)=n ! e_{n}$ since if all $n$ positions have different variables, then they can be permuted in any of $n$ ! ways and still give the same monomial in the projection. In the general case, we have the same phenomenon of multiplication corresponding to shuffling, with each block $B$ contributing $|B|$ !. So the total contribution is $\pi$ !.

Finally we consider (iv). By the same argument as in (iii), it suffices to show that $\rho\left(h_{[n]}\right)=n ! h_{n}$. Consider a monomial $M=x_{j_{1}}^{\lambda_{1}} x_{j_{2}}^{\lambda_{2}} \cdots a_{j_{l}}^{\lambda_{l}}$ in $h_{n}$. These variables can be rearranged to form $n ! / \lambda$ ! monomials in noncommuting variables where $\lambda=$ $\left(\lambda_{1}, \lambda_{2}, \ldots, \lambda_{l}\right)$. To obtain one of these monomials in (3) we must have $\lambda(\operatorname{ker} f)=\lambda$ since $\operatorname{ker} f \wedge[n]=\operatorname{ker} f$. But then the number of pairs $(f, L)$ is just $\lambda$ !. So the number of monomials in $h_{\pi}$ mapping to $M$ under $\rho$ is just $\lambda ! \cdot n ! / \lambda !=n !$, which is what we wanted. 
We end this section by defining a second action of the symmetric group which is also interesting. Since our variables do not commute, we can define an action on places (rather than variables). Explicitly, consider the vector space of elements of $\Pi(\mathbf{x})$ which are homogeneous of degree $n$. Given a monomial of that degree, we define

$$
g \circ\left(x_{i_{1}} x_{i_{2}} \cdots x_{i_{n}}\right)=x_{i_{g(1)}} x_{i_{g(2)}} \cdots x_{i_{g(n)}}
$$

and extend linearly. It is easy to see that if $b_{\pi}$ is a basis element for any of our four bases, then $g \circ b_{\pi}=b_{g \pi}$, where $g$ acts on set partitions in the usual manner.

\section{Change of Basis}

We will now show that all the symmetric functions in noncommuting variables defined in the previous section form bases for $\Pi(\mathbf{x})$. Since we already know this for the $m_{\pi}$, it suffices to find change of basis formulas expressing each function in terms of the $m_{\pi}$ and vice-versa. Doubilet 4 has obtained these results as well as those in the next section in a formal setting that includes ours as a special case. But we replicate his theorems and proofs here for completeness, to present them in standard notation, and to extend and simplify some of them.

Expressing each symmetric function in terms of $m_{\pi}$ is easily done directly from the definitions, so the following proposition is given without proof. In it, all lattice operations refer to $\Pi_{n}$ and $\hat{0}$ is the unique minimal element $1 / 2 / \cdots / n$.

Theorem 3.1. We have the following change of basis formulae:

(i) $p_{\pi}=\sum_{\sigma \geq \pi} m_{\sigma}$,

(ii) $e_{\pi}=\sum_{\sigma \wedge \pi=\hat{0}} m_{\sigma}$

(iii) $h_{\pi}=\sum_{\sigma}(\sigma \wedge \pi) ! m_{\sigma}$.

To express $m_{\pi}$ in terms of the other functions, we will need the Möbius function of the parition lattice $\Pi_{n}$. The Möbius function of any partially ordered set $P$ is the function $\mu: P \times P \rightarrow \mathbb{Z}$ defined inductively by

$$
\mu(a, b)= \begin{cases}1 & \text { if } a=b, \\ -\sum_{a \leq c<b} \mu(a, c) & \text { else. }\end{cases}
$$

This can be rewritten in the useful and more intuitive form

$$
\sum_{a \leq c \leq b} \mu(a, c)=\delta_{a, b}
$$

where $\delta_{a, b}$ is the Kronecker delta. For more information about Möbius functions, see the seminal article of Rota 25] or the book of Stanley [30.

The Möbius function of $\Pi_{n}$ is well known. In particular

$$
\mu(\hat{0}, \hat{1})=(-1)^{n-1}(n-1) !,
$$

where $\hat{1}=12 \cdots n$ is the unique maximal element of $\Pi_{n}$. This is enough to determine $\mu$ on any interval of this lattice. For example, for any $\pi=B_{1} / B_{2} / \cdots / B_{l}$ and 
$\lambda=\lambda(\pi)$ we have the lattice isomorphism $[\hat{0}, \pi] \cong \prod_{i} \Pi_{\lambda_{i}}$. Since the Möbius function is preserved by isomorphism and distributes over products, we have

$$
\mu(\hat{0}, \pi)=\prod_{i}(-1)^{\lambda_{i}-1}\left(\lambda_{i}-1\right) !
$$

Note that, up to sign, this is just the number of permutations $\alpha \in \mathfrak{S}_{n}$ which have disjoint cycle decomposition $\alpha=\alpha_{1} \alpha_{2} \cdots \alpha_{l}$, where, for $1 \leq i \leq l, \alpha_{i}$ is a cyclic permutation of the elements in $B_{i}$. It follows that

$$
\sum_{\sigma \in \Pi_{n}}|\mu(\hat{0}, \sigma)|=n !
$$

Or more generally, because of multiplicativity,

$$
\sum_{\sigma \leq \pi}|\mu(\hat{0}, \sigma)|=\pi !
$$

a result which will be useful shortly. We finally note that if $\sigma=C_{1} / C_{2} / \cdots / C_{m}$ satisfies $\sigma \leq \pi$, then we still have an isomorphism $[\sigma, \pi] \cong \prod_{i} \Pi_{\lambda_{i}(\sigma, \pi)}$, where $\lambda(\sigma, \pi)$ is the integer partition whose $i$ th part is the the number of blocks of $\sigma$ contained in the $i$ th block of $\pi$. (We assume the blocks are listed so that the parts are in weakly decreasing order.) Of course, $\lambda(\hat{0}, \pi)$ is just the type of $\pi$.

The rest of the proofs in this section will all be based on the Möbius Inversion Theorem [25, 30. We will also need a simple corollary of that theorem which slightly generalizes a result of Doubilet [4].

Corollary 3.2. Let $P$ be a poset, let $F$ be a field, and consider three functions $f, g, h: P \rightarrow F$, where $g(a) \neq 0$ for all $a \in P$. Then

$$
\begin{aligned}
& f(a)=\sum_{b \leq a} g(b) \sum_{c \geq b} h(c) \quad \text { for all } a \in P \\
& \Longleftrightarrow h(a)=\sum_{c \geq a} \frac{\mu(a, c)}{g(c)} \sum_{b \leq c} \mu(b, c) f(b) \quad \text { for all } a \in P .
\end{aligned}
$$

Proof. We will prove the forward direction as the converse is obtained by just reversing the steps. Doing (dual) Möbius inversion on the outer sum for $f(a)$ gives

$$
g(a) \sum_{c \geq a} h(c)=\sum_{b \leq a} \mu(b, a) f(b) .
$$

We can divide by $g(a) \neq 0$ and then invert the sum containing $h(c)$, which gives the desired result.

We are now in a position to invert each of the sums in Theorem 3.1 .

Theorem 3.3. We have the following change of basis formulae:

$$
\begin{aligned}
\left(\mathrm{i}^{\prime}\right) m_{\pi} & =\sum_{\sigma \geq \pi} \mu(\pi, \sigma) p_{\sigma}, \\
\left(\mathrm{ii}^{\prime}\right) m_{\pi} & =\sum_{\sigma \geq \pi} \frac{\mu(\pi, \sigma)}{\mu(\hat{0}, \sigma)} \sum_{\tau \leq \sigma} \mu(\tau, \sigma) e_{\tau}, \\
\left(\mathrm{iii}^{\prime}\right) m_{\pi} & =\sum_{\sigma \geq \pi} \frac{\mu(\pi, \sigma)}{|\mu(\hat{0}, \sigma)|} \sum_{\tau \leq \sigma} \mu(\tau, \sigma) h_{\tau} .
\end{aligned}
$$


Proof. Equation (i') follows immediately from the Möbius Inversion Theorem applied to part (i) of Theorem 3.1 .

For identity (ii'), use (66) to write (ii) of Theorem 3.1 in the form

$$
e_{\pi}=\sum_{\sigma}\left(\sum_{\tau \leq \sigma \wedge \pi} \mu(\hat{0}, \tau)\right) m_{\sigma}=\sum_{\tau \leq \pi} \mu(\hat{0}, \tau) \sum_{\sigma \geq \tau} m_{\sigma} .
$$

Using the corollary to invert this double sum gives the desired result.

Finally consider (iii'). Applying (7) to Theorem 3.1(iii) gives

$$
h_{\pi}=\sum_{\sigma}\left(\sum_{\tau \leq \sigma \wedge \pi}|\mu(\hat{0}, \tau)|\right) m_{\sigma}=\sum_{\tau \leq \pi}|\mu(\hat{0}, \tau)| \sum_{\sigma \geq \tau} m_{\sigma} .
$$

The corollary again provides the last step.

The other bases-change equations are derived using similar techniques, so we will content ourselves with merely stating the result after one last bit of notation. We define the sign of $\pi,(-1)^{\pi}$, to be the sign of any permutation obtained by replacing each block of $\pi$ by a cycle. Note that

$$
\mu(\hat{0}, \pi)=(-1)^{\pi}|\mu(\hat{0}, \pi)|
$$

Theorem 3.4. We have the following change of basis formulae:

$$
\begin{aligned}
e_{\pi} & =\sum_{\sigma \leq \pi} \mu(\hat{0}, \sigma) p_{\sigma}, & p_{\pi} & =\frac{1}{\mu(\hat{0}, \pi)} \sum_{\sigma \leq \pi} \mu(\sigma, \pi) e_{\sigma}, \\
h_{\pi} & =\sum_{\sigma \leq \pi}|\mu(\hat{0}, \sigma)| p_{\sigma}, & p_{\pi} & =\frac{1}{|\mu(\hat{0}, \pi)|} \sum_{\sigma \leq \pi} \mu(\sigma, \pi) h_{\sigma}, \\
e_{\pi} & =\sum_{\sigma \leq \pi}(-1)^{\sigma} \lambda(\sigma, \pi) ! h_{\sigma}, & h_{\pi} & =\sum_{\sigma \leq \pi}(-1)^{\sigma} \lambda(\sigma, \pi) ! e_{\sigma} .
\end{aligned}
$$

As an application of these equations, we will derive the properties of an analogue of the involution $\omega: \Lambda(\mathbf{x}) \rightarrow \Lambda(\mathbf{x})$ defined by linearly extending $\omega\left(e_{\lambda}\right)=h_{\lambda}$. Define a map on $\Pi(\mathbf{x})$, which we will also call $\omega$, by $\omega\left(e_{\pi}\right)=h_{\pi}$ for all set partitions $\pi$ and linear extension.

Theorem 3.5. The map $\omega: \Pi(\mathbf{x}) \rightarrow \Pi(\mathbf{x})$ has the following properties:

(i) It is an involution.

(ii) Each $p_{\pi}$ is an eigenvector for $\omega$ with eigenvalue $(-1)^{\pi}$.

(iii) We have $\omega \rho=\rho \omega$.

Proof. (i) It suffices to show that the change of basis matrix between the elementary and complete homogeneous symmetric functions equals its inverse. This follows directly from Theorem 3.4 . 
(ii) We merely compute the action of $\omega$ on the power sum basis by expressing it in terms of the elementary symmetric functions and using equation (8)

$$
\begin{aligned}
\omega\left(p_{\pi}\right) & =\omega\left(\frac{1}{\mu(\hat{0}, \pi)} \sum_{\sigma \leq \pi} \mu(\sigma, \pi) e_{\sigma}\right)=\frac{1}{\mu(\hat{0}, \pi)} \sum_{\sigma \leq \pi} \mu(\sigma, \pi) h_{\sigma} \\
& =\frac{(-1)^{\pi}}{|\mu(0, \pi)|} \sum_{\sigma \leq \pi} \mu(\sigma, \pi) h_{\sigma}=(-1)^{\pi} p_{\pi} .
\end{aligned}
$$

(iii) It suffices to show that the desired equation holds on a basis. So we compute using Theorem 2.1(iii) and (iv): $\omega \rho\left(e_{\pi}\right)=\omega\left(\pi ! e_{\lambda(\pi)}\right)=\pi ! h_{\lambda(\pi)}=\rho\left(h_{\pi}\right)=\rho \omega\left(e_{\pi}\right)$.

\section{The LIFTING MAP AND INNER PRODUCTS}

We will now introduce a right inverse $\tilde{\rho}$ for the projection map $\rho$ and an inner product for which $\tilde{\rho}$ is an isometry. Define the lifting map $\tilde{\rho}: \Lambda(\mathbf{x}) \rightarrow \Pi(\mathbf{x})$ by linearly extending

$$
\tilde{\rho}\left(m_{\lambda}\right)=\frac{\lambda !}{n !} \sum_{\lambda(\pi)=\lambda} m_{\pi}
$$

Proposition 4.1. The map $\rho \tilde{\rho}$ is the identity map on $\Lambda(\mathbf{x})$.

Proof. Equation (4) and Theorem[2.1(i) give $\rho \tilde{\rho}\left(m_{\lambda}\right)=\frac{\lambda !}{n !} \sum_{\lambda(\pi)=\lambda} \lambda^{!} m_{\lambda}=m_{\lambda}$.

Recall that the standard inner product on $\Lambda(\mathbf{x})$ is defined by $\left\langle m_{\lambda}, h_{\mu}\right\rangle=\delta_{\lambda, \mu}$. We define its analogue in $\Pi(\mathbf{x})$ by

$$
\left\langle m_{\pi}, h_{\sigma}\right\rangle=n ! \delta_{\pi, \sigma}
$$

where $\pi \vdash[n]$. This bilinear form respects the grading of $\Pi(\mathbf{x})$ in the sense that if $f, g$ are homogeneous symmetric functions of different degrees, then $\langle f, g\rangle=0$.

Theorem 4.2. The bilinear form $\langle\cdot, \cdot\rangle$ is symmetric, positive definite, and invariant under the action (5).

Proof. For symmetry, it suffices to show that $\left\langle h_{\pi}, h_{\sigma}\right\rangle=\left\langle h_{\sigma}, h_{\pi}\right\rangle$. By Theorem 3.1(iii),

$$
\left\langle h_{\pi}, h_{\sigma}\right\rangle=\left\langle\sum_{\tau}(\pi \wedge \tau) ! m_{\tau}, h_{\sigma}\right\rangle=n !(\pi \wedge \sigma) !
$$

where we let $(\pi \wedge \sigma) !=0$ if $\pi$ and $\sigma$ are partitions of different sets. Noting that $(\pi \wedge \sigma) !=(\sigma \wedge \pi)$ ! completes the proof of symmetry.

As for positive definiteness, take $f \in \Pi(\mathbf{x})$ and write $f=\sum_{\pi} c_{\pi} p_{\pi}$ for certain coefficients $c_{\pi}$. Then, using the expansions for the power sums in Theorems 3.1 
and 3.4, we have

$$
\begin{aligned}
\langle f, f\rangle & =\left\langle\sum_{\sigma} c_{\sigma} p_{\sigma}, \sum_{\tau} c_{\tau} p_{\tau}\right\rangle \\
& =\left\langle\sum_{\sigma} c_{\sigma} \sum_{\pi \geq \sigma} m_{\pi}, \sum_{\tau} c_{\tau} \frac{1}{|\mu(\hat{0}, \tau)|} \sum_{\pi \leq \tau} \mu(\pi, \tau) h_{\pi}\right\rangle \\
& =n ! \sum_{\pi}\left(\sum_{\sigma \leq \pi} c_{\sigma}\right)\left(\sum_{\tau \geq \pi} c_{\tau} \frac{1}{|\mu(\hat{0}, \tau)|} \mu(\pi, \tau)\right) .
\end{aligned}
$$

Now the coefficient of $c_{\sigma} c_{\tau}$ in this last sum is

$$
\frac{n !}{|\mu(\hat{0}, \tau)|} \sum_{\sigma \leq \pi \leq \tau} \mu(\pi, \tau)=\frac{n ! \delta_{\sigma, \tau}}{|\mu(\hat{0}, \tau)|}
$$

Since this is zero for $\sigma \neq \tau$ and positive otherwise, our form is positive definite.

Finally, it suffices to verify invariance under the action on a pair of bases:

$$
\left\langle g \circ m_{\pi}, g \circ h_{\sigma}\right\rangle=\left\langle m_{g \pi}, h_{g \sigma}\right\rangle=n ! \delta_{g \pi, g \sigma}=n ! \delta_{\pi, \sigma}=\left\langle m_{\pi}, h_{\sigma}\right\rangle .
$$

Theorem 4.3. The map $\tilde{\rho}: \Lambda(\mathbf{x}) \rightarrow \Pi(\mathbf{x})$ an isometry, i.e., $\langle f, g\rangle=\langle\tilde{\rho}(f), \tilde{\rho}(g)\rangle$ for $f, g \in \Lambda(\mathbf{x})$.

Proof. It suffices to show that $\left\langle\tilde{\rho}\left(m_{\lambda}\right), \tilde{\rho}\left(h_{\mu}\right)\right\rangle=\left\langle m_{\lambda}, h_{\mu}\right\rangle$ for all $\lambda, \mu$. To compute $\tilde{\rho}\left(h_{\mu}\right)$, consider

$$
H_{\mu}=\sum_{\lambda(\pi)=\mu} h_{\pi}
$$

Expressing $H_{\mu}$ in terms of the monomial symmetric function basis using Theo$\operatorname{rem} 3.1$ (iii), we see that the coefficient of $m_{\sigma}$ is the sum of $(\pi \wedge \sigma)$ ! over all $\lambda(\pi)=\mu$. But the usual action of the symmetric group on set partitions shows that this quantity only depends on $\lambda(\sigma)$. Thus by (9),$H_{\mu}$ must be in the image of $\tilde{\rho}$. Since $\rho$ is a left-inverse for $\tilde{\rho}$, we see that $H_{\mu}$ is the image under $\tilde{\rho}$ of

$$
\rho\left(H_{\mu}\right)=\sum_{\lambda(\pi)=\mu} \rho\left(h_{\pi}\right)=\sum_{\lambda(\pi)=\mu} \lambda(\pi) ! h_{\lambda(\pi)}=\left(\begin{array}{l}
n \\
\mu
\end{array}\right) \mu ! h_{\mu}=\frac{n !}{\mu !} h_{\mu} .
$$

So finally

$$
\begin{aligned}
\left\langle\tilde{\rho}\left(m_{\lambda}\right), \tilde{\rho}\left(h_{\mu}\right)\right\rangle & =\left\langle\frac{\lambda !}{n !} \sum_{\lambda(\pi)=\lambda} m_{\pi}, \frac{\mu^{!}}{n !} \sum_{\lambda(\sigma)=\mu} h_{\sigma}\right\rangle=\frac{\lambda ! \lambda !}{n !^{2}}\left(\begin{array}{c}
n \\
\lambda
\end{array}\right) n ! \delta_{\lambda, \mu} \\
& =\delta_{\lambda, \mu}=\left\langle m_{\lambda}, h_{\mu}\right\rangle .
\end{aligned}
$$

To define the inner product (10) in terms of other pairs of bases, we will need the zeta function of the partition lattice $\Pi_{n}$, defined by

$$
\zeta(\pi, \sigma)= \begin{cases}1 & \text { if } \pi \leq \sigma \\ 0 & \text { else }\end{cases}
$$


Theorem 4.4. The following formulae define equivalent bilinear forms:

$$
\begin{aligned}
\left\langle e_{\pi}, e_{\sigma}\right\rangle & =n !(\pi \wedge \sigma) !, & \left\langle e_{\pi}, h_{\sigma}\right\rangle & =n ! \delta_{\pi \wedge \sigma, \hat{0}}, \\
\left\langle e_{\pi}, p_{\sigma}\right\rangle & =(-1)^{\sigma} n ! \zeta(\sigma, \pi), & \left\langle e_{\pi}, m_{\sigma}\right\rangle & =(-1)^{\sigma} n ! \lambda(\sigma, \pi) ! \zeta(\sigma, \pi), \\
\left\langle h_{\pi}, h_{\sigma}\right\rangle & =n !(\pi \wedge \sigma) !, & \left\langle h_{\pi}, p_{\sigma}\right\rangle & =n ! \zeta(\sigma, \pi), \\
\left\langle h_{\pi}, m_{\sigma}\right\rangle & =n ! \delta_{\pi, \sigma}, & \left\langle p_{\pi}, p_{\sigma}\right\rangle & =n ! \frac{\delta_{\pi, \sigma}}{|\mu(\hat{0}, \pi)|} \\
\left\langle p_{\pi}, m_{\sigma}\right\rangle & =n ! \frac{\mu(\sigma, \pi) \zeta(\sigma, \pi)}{|\mu(\hat{0}, \pi)|}, & \left\langle m_{\pi}, m_{\sigma}\right\rangle & =n ! \sum_{\tau \geq \pi \vee \sigma} \frac{\mu(\pi, \tau) \mu(\sigma, \tau)}{|\mu(\hat{0}, \tau)|}
\end{aligned}
$$

The proof is similar to the derivation of (11), and is omitted.

\section{MacMahon Symmetric FUnCtions}

Schur functions in noncommuting variables will be defined in Section 6, This will require another piece of machinery, namely the MacMahon symmetric functions. The connection between symmetric functions in noncommuting variables and MacMahon symmetric functions was first pointed out by Rosas [23, 24].

Consider $n$ sets, each consisting of a countably infinite number of commuting variables,

$$
\begin{aligned}
\dot{\mathbf{x}} & =\left\{\dot{x}_{1}, \dot{x}_{2}, \ldots\right\}, \\
\ddot{\mathbf{x}} & =\left\{\ddot{x}_{1}, \ddot{x}_{2}, \ldots\right\}, \\
& \vdots \\
\mathbf{x}^{(\mathbf{n})} & =\left\{x_{1}^{(n)}, x_{2}^{(n)}, \ldots\right\} .
\end{aligned}
$$

For each positive integer $m$, the symmetric group $\mathfrak{S}_{m}$ acts on $\mathbb{Q}\left[\left[\dot{\mathbf{x}}, \ddot{\mathbf{x}}, \ldots, \mathbf{x}^{(\mathbf{n})}\right]\right]$ diagonally, i.e.,

$$
g f\left(\dot{x}_{1}, \ddot{x}_{1}, \ldots, \dot{x}_{2}, \ddot{x}_{2}, \ldots\right)=f\left(\dot{x}_{g(1)}, \ddot{x}_{g(1)}, \ldots, \dot{x}_{g(2)}, \ddot{x}_{g(2)}, \ldots\right),
$$

where $g(i)=i$ for $i>m$. We say that $f \in \mathbb{Q}\left[\left[\dot{\mathbf{x}}, \ddot{\mathbf{x}}, \ldots, \mathbf{x}^{(\mathbf{n})}\right]\right]$ is symmetric if it is invariant under the action of $\mathfrak{S}_{m}$ for all $m \geq 1$.

Consider a monomial

$$
M=\dot{x}_{1}^{a_{1}} \ddot{x}_{1}^{b_{1}} \cdots\left(x_{1}^{(n)}\right)^{c_{1}} \dot{x}_{2}^{a_{2}} \ddot{x}_{2}^{b_{2}} \cdots\left(x_{2}^{(n)}\right)^{c_{2}} \cdots .
$$

Letting $\lambda^{i}=\left[a_{i}, b_{i}, \ldots, c_{i}\right]$ be the exponent sequence of the variables of subscript $i$, we define the multiexponent of $M$ to be the vector partition

$$
\vec{\lambda}=\left\{\lambda^{1}, \lambda^{2}, \ldots\right\}=\left\{\left[a_{1}, b_{1}, \ldots, c_{1}\right],\left[a_{2}, b_{2}, \ldots, c_{2}\right], \ldots\right\} .
$$

By summing up the vectors which make up the parts of $\vec{\lambda}$ we get the multidegree of $M$

$$
\vec{m}=\left[m_{1}, m_{2}, \ldots, m_{n}\right]=\left[a_{1}, b_{1}, \ldots, c_{1}\right]+\left[a_{2}, b_{2}, \ldots, c_{2}\right]+\cdots .
$$

In this situation we write $\vec{\lambda} \vdash \vec{m}, \vec{m} \vdash m$, where $m=\sum_{i} m_{i}$, and call $m$ the degree of $M$. We say that $f \in \mathbb{Q}\left[\left[\dot{\mathbf{x}}, \ddot{\mathbf{x}}, \ldots, \mathbf{x}^{(\mathbf{n})}\right]\right]$ has bounded degree if there is a positive integer $m$ such that all monomials in $f$ have degree at most $m$. Define the algebra of MacMahon symmetric functions, $\mathcal{M}=\mathcal{M}\left(\dot{\mathbf{x}}, \ddot{\mathbf{x}}, \ldots, \mathbf{x}^{(\mathbf{n})}\right)$, to be the subalgebra of 
$\mathbb{Q}\left[\left[\dot{\mathbf{x}}, \ddot{\mathbf{x}}, \ldots, \mathbf{x}^{(\mathbf{n})}\right]\right]$ consisting of all $f$ which are symmetric under the action defined by (12) and of bounded degree.

Given a vector partition $\vec{\lambda}$, there is an associated monomial MacMahon symmetric function defined by

$$
m_{\vec{\lambda}}=\text { sum of all the monomials with multiexponent } \vec{\lambda} \text {. }
$$

By way of example,

$$
m_{[2,1],[3,0]}=\dot{x}_{1}^{2} \ddot{x}_{1} \dot{x}_{2}^{3}+\dot{x}_{1}^{3} \dot{x}_{2}^{2} \ddot{x}_{2}+\cdots .
$$

Note that we drop the curly brackets around $\vec{\lambda}$ for readability. These functions are precisely the symmetrizations of monomials in $\mathbb{Q}\left[\left[\dot{\mathbf{x}}, \ddot{\mathbf{x}}, \ldots, \mathbf{x}^{(\mathbf{n})}\right]\right]$ and so they are invariant under the action (12) of $\mathfrak{S}_{m}$ for all $m \geq 1$. It follows easily that they form a basis for $\mathcal{M}$.

Call a basis $b_{\vec{\lambda}}$ of $\mathcal{M}$ multiplicative if it satisfies

$$
b_{\vec{\lambda}}=b_{\lambda^{1}} b_{\lambda^{2}} \cdots b_{\lambda^{l}} .
$$

We now define the bases of power sum, elementary, and complete homogeneous MacMahon symmetric functions to be multiplicative with

$$
\begin{aligned}
p_{[a, b, \ldots, c]} & =m_{[a, b, \ldots, c]}, \\
\sum_{a, b, \ldots, c} e_{[a, b, \ldots, c]} q^{a} r^{b} \cdots s^{c} & =\prod_{i \geq 1}\left(1+\dot{x}_{i} q+\ddot{x}_{i} r+\cdots+x_{i}^{(n)} s\right), \\
\sum_{a, b, \ldots, c} h_{[a, b, \ldots, c]} q^{a} r^{b} \cdots s^{c} & =\prod_{i \geq 1} \frac{1}{1-\dot{x}_{i} q-\ddot{x}_{i} r-\cdots-x_{i}^{(n)} s} .
\end{aligned}
$$

To see the connection with noncommutative symmetric functions, let $\left[1^{n}\right]$ denote the vector of $n$ ones. Now consider the subspace $\mathcal{M}_{\left[1^{n}\right]}$ of $\mathcal{M}$ spanned by all the $m_{\vec{\lambda}}$, where $\vec{\lambda} \vdash\left[1^{n}\right]$. There is a linear map

$$
\Phi: \bigoplus_{n \geq 0} \mathcal{M}_{\left[1^{n}\right]} \rightarrow \Pi
$$

given by

$$
\dot{x}_{i} \ddot{x}_{j} \cdots x_{k}^{(n)} \stackrel{\Phi}{\mapsto} x_{i} x_{j} \cdots x_{k} .
$$

Given any $B \subseteq[n]$, the characteristic vector of $B$ is $\left[b_{1}, b_{2}, \ldots, b_{n}\right]$, where $b_{i}=1$ if $i \in B$ and $b_{i}=0$ otherwise.

Theorem 5.1 (23]). The map $\Phi$ is an isomorphism of vector spaces. Furthermore, for each basis we have discussed

$$
b_{\lambda^{1}, \lambda^{2}, \ldots, \lambda^{l}} \stackrel{\Phi}{\mapsto} b_{B_{1} / B_{2} / \cdots / B_{l}},
$$

where $b=m, p, e$, or $h$, and $\lambda^{i}$ is the characteristic vector of $B_{i}$.

By way of illustration $b_{[1,0,1,0],[0,1,0,1]} \stackrel{\Phi}{\mapsto} b_{13 / 24}$ for any of our bases. 


\section{Schur Functions}

We will now give a combinatorial definition of an analogue of a Schur function in the setting of MacMahon symmetric functions. This will give, via the map $\Phi$, such a function in noncommuting variables. Consider the alphabet

$$
\begin{aligned}
A & =\dot{A} \uplus \ddot{A} \uplus \cdots \uplus A^{(n)} \\
& =\{\dot{1}, \dot{2}, \ldots\} \uplus\{\ddot{1}, \ddot{2}, \ldots\} \uplus \cdots \uplus\left\{1^{(n)}, 2^{(n)}, \ldots\right\} .
\end{aligned}
$$

Partially order $A$ by

$$
i^{(k)}<j^{(l)} \text { if and only if } i<j .
$$

Consider a partition $\lambda$ and a vector $\vec{m}=\left[m_{1}, \ldots, m_{n}\right]$ such that $\lambda, \vec{m} \vdash m$ for some nonnegative integer $m$. Define a dotted Young tableaux $\dot{T}$ of shape $\lambda$ and multidegree $\vec{m}=\left[m_{1}, \ldots, m_{n}\right]$ to be a filling of the shape of $\lambda$ (drawn in English style) with elements of $A$ so that rows are nondecreasing, columns are strictly increasing, and there are $m_{k}$ entries with $k$ dots. Now define the corresponding MacMahon Schur function to be

$$
S_{\lambda}^{\vec{m}}=\sum_{\lambda(\dot{T})=\lambda} M_{\dot{T}} \quad \text { where } \quad M_{\dot{T}}=\prod_{i^{(j)} \in \dot{T}} x_{i}^{(j)}
$$

and the factor $x_{i}^{(j)}$ occurs in the above product with multiplicity, i.e., the same number of times that $i^{(j)}$ occurs in $\dot{T}$. For example, if $\lambda=(3,1)$ and $\vec{m}=[2,2]$, then the coefficient of $\dot{x}_{1}^{2} \ddot{x}_{1} \ddot{x}_{2}$ in $S_{\lambda}^{\vec{m}}$ is 3 , corresponding to the three dotted tableaux

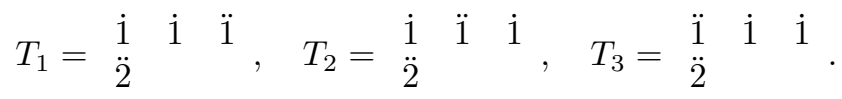

The notion of multidegree generalizes to any multiset $M$ of elements from $A$. If $M$ has $m_{k}$ elements with $k$ dots, we write $\vec{m}(M)=\vec{m}=\left[m_{1}, \ldots, m_{n}\right]$.

Theorem 6.1. The function $S_{\lambda}^{\vec{m}}$ is a MacMahon symmetric function.

Proof. It is obvious that $S_{\lambda}^{\vec{m}}$ is of bounded degree, so we need only show that it is symmetric. Because any permutation is a product of adjacent transpositions, it suffices to show that $S_{\lambda}^{\vec{m}}$ is invariant under the transposition $(i, i+1)$, where $i \geq 1$. So it suffices to find a shape-preserving involution on dotted tableaux $\dot{T} \rightarrow \dot{T}^{\prime}$ which exchanges the number of elements equal to $i^{(k)}$ with the number equal to $(i+1)^{(k)}$ for all $k, 1 \leq k \leq n$. We will use a generalization of a map of Knuth [14 used to prove that the ordinary Schur functions are symmetric.

Since $\dot{T}$ is semistandard, each column contains either a pair $i^{(k)},(i+1)^{(l)}$; exactly one of $i^{(k)}$ or $(i+1)^{(l)}$; or neither. In the first case, replace the pair by $i^{(l)},(i+1)^{(k)}$. In the second, replace $i^{(k)}$ by $(i+1)^{(k)}$ or replace $(i+1)^{(l)}$ by $i^{(l)}$ as appropriate. And in the third case there is nothing to do. It is easy to verify that this involution has the desired properties.

If $\vec{m}=\left[1^{n}\right]$, then we will write $S_{\lambda}$ for $S_{\lambda}^{\vec{m}}$ and make no distinction between $S_{\lambda}$ and its image under the map $\Phi$. The latter will cause no problems because we will never be multiplying these functions. Note also that if $\vec{m}$ has only one component, then $S_{\lambda}^{\vec{m}}=s_{\lambda}$, the ordinary Schur function.

The $S_{\lambda}$ do not form a basis for $\Pi(\mathbf{x})$ since we only have one for every integer, rather than set, partition. However, we can still provide analoques of some of the familiar properties of ordinary Schur functions. To state our results, we will need 
the dominance order on integer partitions, $\mu \unlhd \lambda$, and the Kostka numbers, $K_{\lambda, \mu}$. (For definitions, see [18, 32].)

Theorem 6.2. The functions $S_{\lambda}$ have the following properties:

(i) $S_{\lambda}=\sum_{\mu \unlhd \lambda} \mu ! K_{\lambda, \mu} \sum_{\lambda(\sigma)=\mu} m_{\sigma}$.

(ii) The $S_{\lambda}$ are linearly independent.

(iii) $\rho\left(S_{\lambda}\right)=n ! s_{\lambda}$.

(iv) $\tilde{\rho}\left(n ! s_{\lambda}\right)=S_{\lambda}$.

(v) $\left\langle S_{\lambda}, S_{\mu}\right\rangle=n !^{2} \delta_{\lambda, \mu}$.

Proof. (i) Consider a monomial $x^{\dot{T}}$, where $\dot{T}$ has shape $\lambda$, and suppose that this monomial occurs in $m_{\sigma}$ where $\lambda(\sigma)=\mu$. Then the number of ordinary tableaux $T$ with the same content, $\mu$, as $\dot{T}$ is $K_{\lambda, \mu}$ and this is only nonzero for $\mu \unlhd \lambda$. Furthermore, the number of ways to distribute dots in $T$ so as to give the same monomial as $x^{\dot{T}}$ is $\mu$ !, so this finishes the proof.

(ii) The lexicographic order on integer partitions is a linear extension of the dominance order. So from (i), each $S_{\lambda}$ only contains $m_{\sigma}$ where $\lambda(\sigma)$ is lexicographically less than or equal to $\lambda$, and those with $\lambda(\sigma)=\lambda$ have nonzero coefficient. So if one orders the $S_{\lambda}$ this way, then each Schur function will contain at least one monomial symmetric function not previously found in the list.

(iii) Using (i) again along with Theorem 2.1(i) and (4) gives

$\rho\left(S_{\lambda}\right)=\sum_{\mu \unlhd \lambda} \mu ! K_{\lambda, \mu} \sum_{\lambda(\sigma)=\mu} \rho\left(m_{\sigma}\right)=\sum_{\mu \unlhd \lambda} \mu ! K_{\lambda, \mu} \mu^{!}\left(\begin{array}{l}n \\ \mu\end{array}\right) m_{\mu}=n ! \sum_{\mu \unlhd \lambda} K_{\lambda, \mu} m_{\mu}=n ! s_{\lambda}$.

(iv) Clearly from (i), all $m_{\sigma}$ with $\lambda(\sigma)=\mu$ have the same coefficient in $S_{\lambda}$. So $S_{\lambda}$ is in the image of $\tilde{\rho}$. The equality now follows from (iii) and the fact that $\rho$ is a left-inverse for $\tilde{\rho}$.

(v) We compute using (iv) and the fact that $\tilde{\rho}$ is an isometry

$$
\left\langle S_{\lambda}, S_{\mu}\right\rangle=\left\langle\tilde{\rho}\left(n ! s_{\lambda}\right), \tilde{\rho}\left(n ! s_{\mu}\right)\right\rangle=\left\langle n ! s_{\lambda}, n ! s_{\mu}\right\rangle=n !^{2} \delta_{\lambda, \mu} .
$$

\section{JACOBI-TRUdi DETERMINANTS}

In this section, we prove analogs of the Jacobi-Trudi determinants 18 for the $S_{\lambda}^{\vec{m}}$, where $\vec{m}$ is arbitrary. The ordinary and noncommuting variable cases are obtained as specializations. We use the lattice-path approach introduced by Lindström [16 and developed by Gessel and Viennot [10; see [26] for an exposition.

If $f \in \mathbb{Q}\left[\left[\dot{\mathbf{x}}, \ddot{\mathbf{x}}, \ldots, \mathbf{x}^{(\mathbf{n})}\right]\right]$ and $\vec{m}$ is a vector, then let $\langle\vec{m}\rangle f$ denote the sum of all terms $q_{M} M$ in $f$, where $q_{M} \in \mathbb{Q}$ and $M$ is a monomial of multidegree $\vec{m}$. Also let $\lambda^{\prime}$ denote the conjugate of the partition $\lambda$.

Theorem 7.1. Given a partition $\lambda$ and vector $\vec{m}$ with $\lambda, \vec{m} \vdash m$, we have

$$
S_{\lambda}^{\vec{m}}=\langle\vec{m}\rangle \operatorname{det}\left(\sum_{\vec{t} \vdash \lambda_{i}-i+j} h_{\vec{t}}\right) \quad \text { and } \quad S_{\lambda^{\prime}}^{\vec{m}}=\langle\vec{m}\rangle \operatorname{det}\left(\sum_{\vec{t} \vdash \lambda_{i}-i+j} e_{\vec{t}}\right) \text {. }
$$

Proof. We will only prove the first identity, as the second is obtained by a similar argument. 
Consider infinite paths in the extended integer lattice $\mathbb{Z} \times(\mathbb{Z} \uplus \infty)$ :

$$
P=s_{1}, s_{2}, s_{3}, \ldots
$$

where the $s_{t}$ are steps of unit length either northward $(\mathrm{N})$ or eastward $(\mathrm{E})$. (A point of the form $\left(i^{\prime}, \infty\right)$ can only be reached by ending $P$ with an infinite number of northward steps along the line $x=i^{\prime}$.) If $P$ starts at $(i, j)$, then we label an eastward step along the line $y=j^{\prime}$ with the label

$$
L\left(s_{t}\right)=\left(j^{\prime}-j+1\right)^{(k)}
$$

for some $k$ which can vary with the step, $1 \leq k \leq n$. Considering $P$ as a multiset of labels, it has a well-defined multidegree $\vec{t}$. Then for $\vec{t} \vdash t$ we have

$$
h_{\vec{t}}=\sum_{P} M_{P} \quad \text { where } \quad M_{P}=\prod_{\ell^{(k)}} x_{\ell}^{(k)},
$$

the sum being over all paths of multidegree $\vec{t}$ from $(i, j)$ to $(i+t, \infty)$ and the product being over all labels in $P$ taken with multiplicity. Note also that if the labels in $P$ are read off from left to right, then they correspond to a single-rowed dotted Young tableau of multidegree $\vec{t}$.

To get products of complete homogeneous symmetric functions and tableaux of shape $\lambda=\left(\lambda_{1}, \ldots, \lambda_{l}\right)$, consider initial vertices $u_{1}, \ldots, u_{l}$ and final vertices $v_{1}, \ldots, v_{l}$ with coordinates

$$
u_{i}=(-i, 1) \quad \text { and } \quad v_{i}=\left(\lambda_{i}-i, \infty\right)
$$

for $1 \leq i \leq l$. Consider a family of labeled paths $\mathcal{P}=\left(P_{1}, \ldots, P_{l}\right)$, where, for each $i, P_{i}$ is a path from $u_{i}$ to $v_{g(i)}$ for some $g \in \mathfrak{S}_{l}$. We assign to $\mathcal{P}$ a monomial and a sign by

$$
M_{\mathcal{P}}=\prod_{i=1}^{l} M_{P} \quad \text { and } \quad(-1)^{\mathcal{P}}=(-1)^{g},
$$

respectively. So denoting the determinant by $D$, we have

$$
D=\sum_{\mathcal{P}}(-1)^{\mathcal{P}} M_{\mathcal{P}}
$$

where the sum is over all path families with beginning and ending vertices given by (14).

Construct a monomial-preserving, sign-reversing involution $\imath$ on such $\mathcal{P}$ which are intersecting as follows. Let $i$ be the smallest index such that $P_{i}$ intersects some $P_{j}$ and take $j$ minimum. Consider the NE-most point, $v_{0}$, of $P_{i} \cap P_{j}$. Create $\mathcal{P}^{\prime}=\imath \mathcal{P}$ by replacing $P_{i}, P_{j}$ with $P_{i}^{\prime}, P_{j}^{\prime}$, respectively, where $P_{i}^{\prime}$ goes from $u_{i}$ to $v_{0}$ along $P_{i}$ and then continues along $P_{j}$, and similarly for $P_{j}^{\prime}$.

Because $\imath$ pairs up intersecting path families of the same monomial and opposite sign, they all cancel from the determinant leaving only nonintersecting families. Furthermore, by the choice of initial and final points, a family can only be nonintersecting if its associated element of $\mathfrak{S}_{l}$ is the identity. So we now have

$$
D=\sum_{\mathcal{P}} M_{\mathcal{P}}
$$

where the sum is over all nonintersecting families. But there is a bijection between such families and tableaux. Given $\mathcal{P}$, read the elements of $P_{i}$ from left to right to obtain the $i$ th row of the associated tableau $\dot{T}$. The fact that $\mathcal{P}$ is nonintersecting is equivalent to the fact that $\dot{T}$ has increasing columns. The given initial and final 
vertices ensure that the shape of $\dot{T}$ is $\lambda$. Applying $\langle\vec{m}\rangle$ to both sides of the last equality restricts the multidegree so as to finish the proof of the theorem.

Note that if $\vec{t}=[t]$ has a single component, then $\vec{t} \vdash \lambda_{i}-i+j$ forces $t=\lambda_{i}-i+j$. So the sums in each entry of the determinants reduce to a single term and we recover the ordinary form of Jacobi-Trudi.

We now specialize to the case of noncommuting variables case so as to determine the image of $S_{\lambda}$ under the involution $\omega$.

Corollary 7.2. We have $\omega\left(S_{\lambda}\right)=S_{\lambda^{\prime}}$.

Proof. Merely note that $\omega$ exchanges the two Jacobi-Trudi determinants.

\section{The Robinson-Schensted-Knuth MaP}

In this section, we give a generalization of the famous Robinson-Schensted-Knuth bijection [14, 22, 27] to tableaux of arbitrary multidegree.

A biword of length $n$ over $A$ is a $2 \times n$ array $\beta$ of elements of $A$ such that if the dots are removed, then the columns are ordered lexicographically with the top row taking precedence. The lower and upper rows of $\beta$ are denoted $\breve{\beta}$ and $\hat{\beta}$, respectively. Viewing $\breve{\beta}$ and $\hat{\beta}$ as multisets, the multidegree of $\beta$ is the pair

$$
\vec{m}(\beta)=(\vec{m}(\check{\beta}), \vec{m}(\hat{\beta})) .
$$

We now define a map $\beta \stackrel{\mathrm{R}-\mathrm{S}-\mathrm{K}}{\mapsto}(\dot{T}, \dot{U})$ whose image is all pairs of dotted semistandard Young Tableaux of the same shape. Peform the ordinary Robinson-SchenstedKnuth algorithm on $\beta$ (see 26 for an exposition) by merely ignoring the dots and just having them "come along for the ride." For example, if

$$
\beta=\begin{array}{llllll}
\dot{1} & \dot{2} & \ddot{2} & \dot{2} & \ddot{3} & \ddot{4} \\
\dot{2} & \ddot{1} & \ddot{3} & \dot{3} & \ddot{2} & \dot{1},
\end{array}
$$

then the sequence of tableaux built by the algorithm is

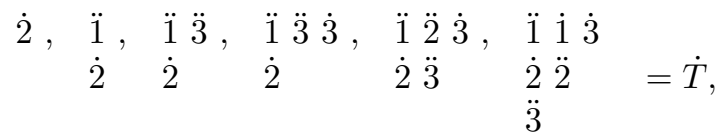

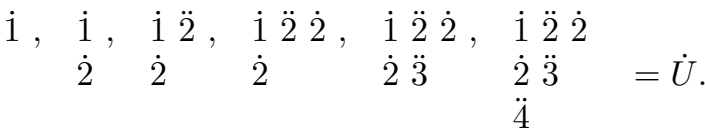

The next theorem follows directly from the definitions and the analogous result for the ordinary Robinson-Schensted-Knuth map.

Theorem 8.1. The map

$$
\beta^{\mathrm{R}-\mathrm{S}-\mathrm{K}}(\dot{T}, \dot{U})
$$

is a bijection between biwords and pairs of dotted semistandard Young tableaux of the same shape such that

$$
\vec{m}(\beta)=(\vec{m}(\dot{T}), \vec{m}(\dot{U})) .
$$


Because this analogue is so like the original, most of the properties of the ordinary Robinson-Schensted-Knuth correspondence carry over into this setting with virtually no change. By way of illustration, here is the corresponding Cauchy identity [17] which follows directly by turning each side of the previous bijection into a generating function. Note that for $\hat{\beta}$ and $\dot{U}$ we are using a second set of variables $\dot{\mathbf{y}}, \ddot{\mathbf{y}}, \ldots, \mathbf{y}^{(\mathbf{n})}$.

Theorem 8.2. We have

$$
\sum_{m \geq 0} \sum_{\lambda, \vec{m}, \vec{p} \vdash m} S_{\lambda}^{\vec{m}}\left(\dot{\mathbf{x}}, \ldots, \mathbf{x}^{(\mathbf{n})}\right) S_{\lambda}^{\vec{p}}\left(\dot{\mathbf{y}}, \ldots, \mathbf{y}^{(\mathbf{n})}\right)=\prod_{i, j \geq 1} \frac{1}{1-\sum_{k, l=1}^{n} x_{i}^{(k)} y_{j}^{(l)}} .
$$

\section{Comments And questions}

(I) Rosas [24] computed specializations of symmetric functions in noncommuting variables and, more generally, of MacMahon symmetric functions.

(II) Is there an expression for $S_{\lambda}^{\vec{m}}$ analogous to Jacobi's bialternant formula [18, 26, 32]?

(III) Is there a connection between $\Pi(\mathbf{x})$ and the partition algebra $P_{n}(x)$, 11, 12, 20]?

(IV) Is there a way to define functions $S_{\pi}$ for set partitions $\pi \vdash[n]$ having properties analogous to the ordinary Schur functions $s_{\lambda}$ ?

(V) Given a basis $b_{\lambda}(\mathbf{x})$ for $\Lambda(\mathbf{x})$ we say that $f(\mathbf{x}) \in \Lambda(\mathbf{x})$ is b-positive if the coefficients in the expansion $f(\mathbf{x})=\sum_{\lambda} c_{\lambda} b_{\lambda}(\mathbf{x})$ satisfy $c_{\lambda} \geq 0$ for all $\lambda$. Stanley [29, 31. showed that associated with any combinatorial graph $G$ there is a symmetric function $X_{G}(\mathbf{x}) \in \Lambda(\mathbf{x})$ which generalizes the chromatic polynomial of $G$. Together with Stembridge [33, he conjectured that for a certain family $\mathcal{G}$ of graphs (those associated with $(\mathbf{3}+\mathbf{1})$-free posets) $X_{G}$ is $e$-positive for all $G \in \mathcal{G}$. Gasharov [7] has proved the weaker result that $X_{G}$ is $s$-positive for all $G \in \mathcal{G}$. Gebhard and Sagan [8] have proved that $X_{G}$ is $e$-positive for all $G$ in a subfamily of $\mathcal{G}$ by using symmetric functions in noncommuting variables. It would be interesting to enlarge the subfamily to which these methods can be applied.

\section{ACKNOWLEDGMENTS}

Part of the research for this paper was done while both authors were residents at the Isaac Newton Institute for Mathematical Sciences in Cambridge, England. We thank the Institute for support during this period. We are also indebted to Timothy Chow, Ira Gessel, and Larry Smith for helpful discussions. Finally, we owe a great debt of thanks to an anonymous referee whose incredibly detailed and helpful comments greatly improved the exposition.

\section{REFERENCES}

[1] D. J. Anick, On the homogeneous invariants of a tensor algebra, in "Algebraic Topology: Proceedings of the International Conference held March 21-24, 1988," Mark Mahowald and Stewart Priddy eds., Contemporary Mathematics, Vol. 96, American Math. Society, Providence, RI, 1989, 15-17. MR90i:55033

[2] G. M. Bergman and P. M. Cohn, Symmetric elements in free powers of rings, J. London Math. Soc. (2) 1 (1969), 525-534. MR40:4301

[3] W. Doran and D. Wales, The partition algebra revisited, J. Algebra 231 (2000), 265-330. MR2001i:16032 
[4] P. Doubilet, On the foundations of combinatorial theory. VII: Symmetric functions through the theory of distribution and occupancy, Studies in Applied Math. 51 (1972), 377-396. MR.55:2589

[5] S. Fomin and C. Greene, Noncommutative Schur functions and their applications, Discrete Math. 193 (1998), 179-200. MR2000c:05149

[6] W. Fulton, "Young Tableaux," London Mathematical Society Student Texts 35, Cambridge University Press, Cambridge, 1999. MR99f:05119

[7] V. Gasharov, Incomparability graphs of $(\mathbf{3}+\mathbf{1})$-free posets are s-positive, Discrete Math. 157 (1996), 193-197. MR98k:05140

[8] D. Gebhard and B. Sagan, A chromatic symmetric function in noncommuting variables, $J$. Algebraic Combin. 13 (2001), 227-255. MR2002d:05124

[9] I. M. Gelfand, D. Krob, A. Lascoux, B. Leclerc, V. Retakh, J.-I. Thibon, Noncommutative symmetric functions, Adv. in Math. 112 (1995), 218-348. MR96e:05175

[10] I. Gessel and G. Viennot, Binomial determinants, paths, and hook length formulae, Adv. in Math. 58 (1985), 300-321. MR87e:05008

[11] T. Halverson, Characters of the partition algebra, J. Algebra 238 (2001), 502-533. MR2002a:20019

[12] T. Halverson and J. Fraina, Character orthogonality for the partition algebra and fixed points of permutations. Adv. Appl. Math. 31 (2003), 113-131. MR2004e:16016

[13] V. K. Kharchenko, Algebras of invariants of free algebras, Algebra i Logika 17 (1978) 478-487 (Russian); Algebra and Logic 17 (1978), 316-321 (English translation). MR80e:16003

[14] D. E. Knuth, Permutations, matrices and generalized Young tableaux, Pacific J. Math. 34 (1970), 709-727. MR 42:7535

[15] A. Lascoux and M.-P. Schützenberger, Le monoid plaxique, in "Noncommutative Structures in Algebra and Geometric Combinatorics, (Naples, 1978)," Quad. Ricerca Sci., Vol. 109, CNR, Rome, 1981, 129-156. MR83g:20016

[16] B. Lindström, On the vector representation of induced matroids, Bull. London Math. Soc. 5 (1973), 85-90. MR 49:95

[17] D. E. Littlewood, "The Theory of Group Characters," Oxford University Press, Oxford, 1950. MR2:3a

[18] I. G. Macdonald, "Symmetric functions and Hall polynomials," 2nd edition, Oxford University Press, Oxford, 1995. MR96h:05207

[19] P. A. MacMahon, "Combinatorial Analysis," Vols. 1 and 2, Cambridge University Press, Cambridge, 1915, 1916; reprinted by Chelsea, New York, NY, 1960.

[20] P. Martin, The structure of partition algebras, J. Algebra 183 (1996), 319-358. MR98g:05152

[21] S. D. Noble and D. J. A. Welsh, A weighted graph polynomial from chromatic invariants of knots, Symposium à la Mémoire de François Jaeger (Grenoble, 1998) Annales l'Institut Fourier 49 (1999), 1057-1087. MR2000h:05066

[22] G. de B. Robinson, On representations of the symmetric group, Amer. J. Math. 60 (1934), $745-760$.

[23] M. H. Rosas, MacMahon symmetric functions, the partition lattice, and Young subgroups, J. Combin. Theory Ser. A 96 (2001), 326-340. MR2002k:05241

[24] M. H. Rosas, Specializations of MacMahon symmetric functions and the polynomial algebra, Discrete Math. 246 (2002), 285-293. MR2003h:05189

[25] G.-C. Rota, On the foundations of combinatorial theory I. Theory of Möbius functions, $Z$. Wahrscheinlichkeitstheorie 2 (1964), 340-368. MR.30:4688

[26] B. Sagan, "The Symmetric Group: Representations, Combinatorial Algorithms, and Symmetric Functions," 2nd edition, Springer-Verlag, New York, 2001. MR2001m:05261

[27] C. Schensted, Longest increasing and decreasing subsequences, Canad. J. Math. 13 (1961), 179-191. MR22:12047

[28] I. Schur, "Über eine Klasse von Matrizen die sich einer gegebenen Matrix zuordnen lassen," Inaugural-Dissertation, Berlin, 1901.

[29] R. P. Stanley, A symmetric function generalization of the chromatic polynomial of a graph, Advances in Math. 111 (1995), 166-194. MR96b:05174

[30] R. P. Stanley, "Enumerative Combinatorics, Volume 1," Cambridge University Press, Cambridge, 1997. MR98a:05001 
[31] R. P. Stanley, Graph colorings and related symmetric functions: ideas and applications: A description of results, interesting applications, \& notable open problems, Selected papers in honor of Adriano Garsia (Taormina, 1994) Discrete Math. 193 (1998), 267-286. MR2000c:05152

[32] R. P. Stanley, "Enumerative Combinatorics, Volume 2," Cambridge University Press, Cambridge, 1999. MR2000k:05026

[33] R. P. Stanley and J. Stembridge, On immanants of Jacobi-Trudi matrices and permutations with restricted position, J. Combin. Theory Ser. A 62 (1993), 261-279. MR94d:05147

[34] L. Weisner, Abstract theory of inversion of finite series, Trans. Amer. Math. Soc. 38 (1935), 474-484.

[35] H. Whitney, A logical expansion in mathematics, Bull. Amer. Math. Soc. 38 (1932), 572-579.

[36] M. C. Wolf, Symmetric functions of noncommuting elements, Duke Math. J. 2 (1936), 626637.

Departamento de Matemáticas, Universidad Simón Bolívar, Apdo. Postal 89000, Caracas, Venezuela

E-mail address: mrosas@ma.usb.ve

Current address: Department of Mathematics \& Statistics, York University, Toronto, Ontario, Canada M3J 1P3

Department of Mathematics, Michigan State University, East Lansing, Michigan 48824-1027

E-mail address: sagan@math.msu.edu 